\title{
Standard circumstances and vital goals: comments on Sridhar Venkatapuram's critique
}

\author{
Lennart Nordenfelt
}

\section{Linköping University Post Print}

\section{Tweet}

N.B.: When citing this work, cite the original article.

This is the pre-reviewed version of the following article:

Lennart Nordenfelt , Standard circumstances and vital goals: comments on Sridhar Venkatapuram's critique, 2013, Bioethics, (27), 5, 280-284.

which has been published in final form at:

http://dx.doi.org/10.1111/j.1467-8519.2011.01958.x

Copyright: Wiley-Blackwell

http://eu.wiley.com/WileyCDA/Brand/id-35.html

Postprint available at: Linköping University Electronic Press

http://urn.kb.se/resolve?urn=urn:nbn:se:liu:diva-77992 


\title{
Standard circumstances and vital goals: Comments on Sridhar Venkatapuram's critique
}

\author{
Lennart Nordenfelt \\ Dept of Medical and Health Sciences \\ Linköping University \\ 58183 Linköping
}

Sweden

e-mail: lennart.nordenfelt@liu.se

\section{Introduction}

It was very encouraging and intriguing to read Dr. Venkatapuram's comparative analysis of my own theory of the concept of health and its related concepts (1995; 2000 and 2007) and Professor Martha Nussbaum's very renowned theory of capabilities as conditions for human flourishing (2006 and 2011). I am pleased to see that there are crucial relations between the two theories and I can envisage it as being a very interesting task to develop Venkatapuram's comparison. However, this must wait. In my present reply to Venkatapuram's article I will concentrate on his particular critique of my own views.

Let me as a starting point use the present formulation of my definition of health:

$\mathrm{A}$ is completely healthy, if and only if $\mathrm{A}$ is in a bodily or mental state which is such that $\mathrm{A}$ has the second-order ability to realize all his or her vital goals given standard or reasonable circumstances. The set of a A's vital goals is the set of states of affairs which are necessary and together sufficient for A's minimal happiness in the long run.

I will first comment on the set of circumstances mentioned in the definition. I have worked with two kinds of specifications that have a place in two different contexts. On the one hand, I assume a set of standard circumstances, a set commonly presupposed within a culture, as is done within a clinical context. When we judge a person to be ill we normally use a standard implicit in our society. When we say that a Swede is able to walk around we (including the doctor) are presupposing that the walking is to be performed given standard Swedish 
circumstances, for instance the kind of ground we have outdoors, the kind of climate we normally have, excluding the occasional storms and excluding perhaps the extreme temperatures. We also presuppose that the political circumstances are not extreme, that the country is not at war and thereby highly restricting people's movements. Moreover, we presuppose that the person is not imprisoned and for that reason not completely prevented from moving about freely.

For other discourses I assume a set of reasonable circumstances. The term "reasonable" indicates that we are dealing with a more openly normative discourse. A paradigmatic context would be where a language-user openly disputes an illness ascription. "You call this person ill; no, she is perfectly healthy, she is only prevented from acting by the unreasonable circumstances in which she is placed." We can imagine that the person lives in a country which is continuously in a precarious situation, i.e. where in a sense the standard circumstances are continuously unreasonable. It is easy to find such examples today, in Haiti, in Afghanistan and Libya. A teacher in one of these countries may be continuously prevented from executing her profession for the simple reason that there is no functioning school in her area. It would however be absurd to say that this person is unhealthy because she cannot execute her profession in the present standard situation. This person is perfectly healthy even though she is disabled from doing what could normally be expected from her. To capture both the sense of standard and of reasonable circumstances, I have later coined the term "accepted" circumstances (for instance Nordenfelt 2000). In every health discourse, some set of circumstances must be accepted. In some discourses we accept a set of culturally defined standard circumstances. In other discourses, we stipulate a set of reasonable circumstances. In a way I propose therefore both a descriptive and a normative version of the concept of health.

A major point illustrated in my definition is that I sharply separate ability from the external circumstances. Ability to do F is not enough for actually doing F. There must be favourable circumstances and there must of course also be a will to do F. Ability plus a favourable circumstance (i.e. an opportunity) form together what I call a practical possibility. (Nussbaum 2011 proposes the term "combined capability" for roughly the same concept.)

It is then clear that a healthy person, in my interpretation, who is able to do F, given standard or reasonable circumstances, need not, at a particular moment, succeed in doing F even though she wants to. She may not, at this particular moment, have a practical possibility of 
doing F; there may be obstacles of various kinds in her way, i.e. there may at this moment be non-standard or unreasonable circumstances. Therefore, it is the (internal) ability that is at stake in my health definition, not the practical possibility.

\section{The relation between circumstances and vital goals}

Let me then turn to one of Venkatapuram's major critical points. He says that by relying on standard or even reasonable circumstances I make vital goals relative to each community or context. He says that the circumstance clause in my definition of health entails a "complete capitulation to the local social circumstances in determining the content of vital goals. This exposes Nordenfelt's theory to the charge of advocating social and ethical relativism." He goes on to say: "A theory of health is needed precisely to specify or evaluate rights and obligations related to health where local circumstances conflict with or fall well below what are considered to be reasonable circumstances (pp. 11-12).”

I think there is a misunderstanding here which I wish to resolve. I have not intended that there should be a conceptual connection between standard circumstances and vital goals at all. Besides, I wonder how any ability-based theory of health can avoid a notion similar to my standard or reasonable circumstances. When one assesses that a person has a certain ability one cannot do that in a vacuum, one must presuppose some background. In a clinical context, for instance, one normally presupposes some standard circumstances, excluding the extreme ones. Occupational therapists do not judge a person's ability to walk when the person tries to walk in a snowstorm, uphill Kilimanjaro or through a battle zone. They judge the ability against the background of standard or reasonable circumstances such as mild weather, normal exertion and peaceable surroundings.

The standard or reasonable circumstances, then, specify the platform for the person's ability. The person's vital goals, however, are in principle completely unconnected to the circumstances. The local or social circumstances do not "determine the content of vital goals", as Venkatapuram would have it. A person may have several vital goals which have nothing to do with the local culture. A person living in a very poor society may sincerely aspire to a position in life which she knows can only be realized in a different society. In such a case it would be absurd to say that the vital goal is determined by the local culture. 
On the other hand, Venkatapuram may argue that even though there are no conceptual relations between my notions of standard circumstance and vital goals there may be crucial empirical relations. A person who lives in quite a closed society with few or no possibilities of learning what options exist outside that society cannot really aspire for or desire much that exists out there. If one does not learn at school or at home that there are other kinds of lives to live then one cannot long for these lives. Therefore, it would seem as if the standard circumstances in a way restrict and partly determine the formation of the person's vital goals.

My answer is that the circumstances may restrict the person's conscious wants and desires. But we must not forget that my notion of a vital goal is a theoretical notion not tied to a person's present conscious wants and beliefs. A vital goal of $\mathrm{P}$ is defined as a state of affairs which is a necessary condition for P's long-term minimal happiness. Often a vital goal of an (adult) person is identical with what she sincerely and deeply wants, but it need not be. (For one thing I cannot connect vital goals to conscious wants in my theory since also babies and demented persons have vital goals.) And in the case of a person who is ignorant of most goods in life her vital goals are not identical with the objects of her conscious wants. The poverty-stricken person in the Central African Republic would probably have a happier life in northern Europe than in her home-country. Among this person's vital goals are therefore some of which she may not be aware. Thus, also the empirical links between standard circumstances and vital goals appear to be weak.

Perhaps another, more subtle, empirical link exists. There may be some truth in the idea that a culture does not only influence a person's conscious wants and beliefs. The culture may also in the end influence this person's ability to flourish and experience happiness. The person may be so completely accustomed to and tied to a form of life that she cannot really leave it behind and appreciate something completely new. I can imagine that this might be the case and consequently I do not wish to rule out this empirical connection between circumstances and vital goals.

\section{The content of the set of vital goals}

I will now deal with a second critique formulated by Venkatapuram. He says that I stop short of explicitly filling in the framework of vital goals with any content (pp.9-10). I do not present a list of vital goals common to mankind that would make my concept of health much more concrete. Venkatapuram then contrasts my "empty set" of vital goals with Martha 
Nussbaum's ingenious list of ten basic human capabilities worthy of human dignity (Nussbaum 2006 and 2011). I fail to do something important, Venkatapuram says. My theory must be filled with content.

I think this is an interesting critique and I think that it is worth analysing. It is true that I have a (fairly) empty set of vital goals in my general characterization of the notion. But I give some more concrete hints, as Venkatapuram notes, in the following: "Being alive is a necessary condition of being happy... Hence all the necessary conditions for the maintenance of life must be included among every person's vital goals, for instance having food, having a sheltered home and having some economic security" (Nordenfelt, 1995, p. 91). From these fundamental vital goals we can certainly deduce much more. In most societies, going to work and doing one's job is a necessary condition for having economic security. Thus, doing one's job must normally be a vital goal to all (at least Western) adults before their retirement.

However, it is true that the fundamental vital goals and their logical consequences do not exhaust any person's complete set of vital goals. Living a life in minimal happiness is certainly something more than just living.

Yes, I have consciously chosen to stop short of explicitly filling the set of vital goals. The main reason is that I think that human beings are to a greater extent different from each other than Venkatapuram assumes. Let us just consider the fact that people have very different jobs. Some people have manual jobs, like servants, carpenters and masons; others have artistic jobs, like painters and pop singers; others are professional athletes, like tennis players and golfers; yet others have intellectual jobs, like bureaucrats and teachers. Most people are positively engaged in their particular jobs. Thus, most of these professionals have some of their vital goals connected to their jobs. This partly means that they would become deeply depressed if they could not pursue them. The servant who breaks an arm faces a personal disaster; for the carpenter and some athletes it would be equally bad. For the ordinary teacher and bureaucrat, however, the breaking of an arm need not have much negative influence at all. Conversely, certain cognitive defects would be more fatal to a person with an intellectual job than to others. An economist who has lost her proficiency in calculating costs is quite disabled. A carpenter with a similar defect is not equally disabled. 
This means that the servant with a broken arm has a lower degree of health than the teacher with an identical broken arm. The ability to carry things around in a smooth way is a vital goal to the servant but not to the teacher. This has consequences for actual health care at least in Western countries. In an ambitious clinic one would rehabilitate a servant in a different way and provide more specific training for her than one would for other persons with a similarly broken arm.

Thus, I contend that people have partly quite individual vital goals that must be considered in the analysis of their health states. This does not only hold with regard to their professions but in general to their different interests. I therefore abstain from specifying universal vital goals in any concrete way. (One might perhaps find very abstract characterizations by talking in general terms about people's aims and interests, but I am not sure this is what Venkatapuram asks for.)

How do I then react to Martha Nussbaum's list of basic human capabilities? Are the objects of those capabilities candidates for being vital goals in my sense? I envisage a fascinating project to analyse this. In this context I can only make some preliminary remarks.

First, Nussbaum and I have different aims. She wants to characterize a dignified and flourishing life; I want to characterize health. A major theoretical difference between us therefore is that she does not just talk about (internal) ability in my sense but about a person's practical possibility (or "combined capability" in her terminology). The flourishing life presupposes not just the internal strength of a person but also that there are favourable circumstances. Nussbaum's (and the whole capability movement's) purpose is indeed to a high degree political in underlining the duties of societies to provide supportive environments for people to flourish.

The difference in our projects becomes particularly visible in Nussbaum's formulation of the second capability in her list, viz. the one regarding bodily health: "Being able to have good health, including reproductive health" (Nussbaum 2011, p. 33). Health cannot for logical reasons be a vital goal of the ability entailed in health. (But perhaps we could avoid the vicious circle if we mean the maintenance of health and not merely the acquisition of health.) Certain other capabilities quite explicitly concern the external conditions and must therefore lie outside my vital goals. Among these are elements in capability 10: "Having the right of 
political participation, protections of free speech and association" and "Being able to hold property ... and having property rights on an equal basis with others" (Nussbaum 2011, p. 34).

Otherwise, I find that several of the objects of Nussbaum's capabilities are quite good candidates for being vital goals. Indeed, some of them are candidates for being fundamental vital goals (i.e. universal goals in the sense of being necessary for survival). This is so with most elements in capability 3 on Bodily integrity and capability 4 on Senses, imagination, and thought. Being able to move freely from place to place and being able to use one's senses, to imagine, think and reason to a certain extent, are very basic abilities that are required for the performance of most conceivable actions including those which are needed for mere survival.

There are, however, such capabilities in Nussbaum's list as are not necessarily included in everybody's set of vital goals. I doubt whether it is reasonable to say that all healthy people must be able to form a conception of the good and engage in critical reflection about the planning of one's life (Capability 6 Practical reason). It seems here as if Nussbaum requires that a dignified person has both moral sensitivity and considerable intelligence. This may hold for the dignified person but not necessarily for the ordinary healthy person. One might also discuss to what extent the objects of capability 8 (Other species) and 9 (Play) are universal vital goals. Is having a concern for animals and plants necessary for the minimal happiness of all people? And must all healthy people be able to play?

I do not here wish to be misunderstood. I certainly think that all Nussbaum's capabilities are in general desirable capabilities. I can sympathize with Nussbaum's anthropology and I can on the whole agree with her idea about what is required for a dignified life. But to repeat: my focus of study (in this context) has been on human health and not on what is a dignified life. (I have, however, in a different context (Nordenfelt 2009) paid much attention to the concept of dignity.)

\section{The definition of vital goals in relation to our intuitions about health}

Let me now turn to Venkatapuram's third critique (p.17). He borrows a critical commentary formulated by Thomas Schramme (2007). Schramme argues that my definition of vital goals is too broad. Schramme's example concerns a young athlete Lily, who is a high jumper. Lily has struggled for a long time to become an accomplished athlete, but she has not succeeded 
and is very unhappy. Since Lily has thus not achieved her minimal happiness, according to my theory, she is not healthy. Schramme thinks that this is an odd conclusion, especially since Lily has no disease.

As Venkatapuram says I have already answered this critique (Nordenfelt 2007). I noted that Schramme here presupposes that health and illness are contradictory terms in the sense that wherever we do not have complete health we have illness. In my understanding health is a dimension ranging from a state of complete health to a state of maximal illness. So, when Lily does not achieve what she has striven for so intensely, and this goal qualifies as a vital goal in my sense, the assessment is not automatically that Lily is ill. The result is only that Lily's complete health is somewhat reduced. Presumably, Lily is in the example able to realize most if not all other vital goals, in particular what I call fundamental vital goals, i.e. such goals as are required for Lily's survival.

Presumably Schramme and Venkatapuram would not even admit that Lily's health is somewhat reduced. She is, they might maintain, plainly healthy. I then wish to underline something that Venkatapuram in general accepts - that a non-ability to achieve a vital goal need not be due to an obvious disease (in the traditional sense of the word). It could be due to an unrealistic goal. There is a mismatch between Lily's abilities and her goals that causes her a lot of unhappiness. This mismatch is attributable to Lily's completely unrealistic ambitions. So, if we consider that Lily should be helped in this instance, the cure is not to turn to orthopaedics but rather to psychotherapy. The cure lies in what could be called goal-care. We should try to convince Lily of the unrealistic nature of her goal, and convince her that she should not strive for this goal in this intense way. Of course she could very well set a high, or even utopian, goal but then she must be emotionally prepared for the fact that this goal is not going to be realized. Thus, her health need not be affected by her failure.

Venkatapuram is not satisfied with my answer, however. He claims that for a health-scale from optimal health to maximal illness to work one must create thresholds between optimal health and moderate health, as well as between moderate health and ill health. And, he says, it is unclear where or how these thresholds will be created.

I agree that we must provide clear thresholds in order to make these distinctions when we operationalize the health concept in clinical situations, for instance for the purpose of sick- 
listing people. It seems to me unreasonable, however, to expect that I should in the basic philosophical analysis give clear definitions of such thresholds. Does Venkatapuram formulate similar requirements with regard to the thresholds entailed in Nussbaum's theory of capabilities?

\section{The relation between health and subjective preferences}

Venkatapuram finally remarks that I might let a person's subjective preferences or ideas of happiness determine health too much (p. 18). Again, this contention of his has to be qualified. As I noted above there is not a one-to-one relationship between the objects of a person's subjective preferences or wants, on the one hand, and her vital goals, on the other. Health in my understanding is not dependent on a person's present conscious wants or desires. Nor is it dependent on a person's happiness, or even tendency to be happy, at a particular moment. A healthy person can be unhappy for a number of external reasons, for instance because she has lost her job, and an unhealthy person can be extremely happy, for instance because of favourable financial prospects or the success of her children. It should again be emphasized that health, in my understanding, is primarily an ability-concept, viz. the ability to achieve vital goals.

It is correct, however, that the criterion of the realization of a subject's all vital goals is in the end that the subject is minimally happy (in an emotional sense) in the long run. This means, among other things, that the "objective" physiological background of two healthy persons can be different, and, conversely, the physiological background can be exactly the same of two unhealthy persons.

It is also true, then, that for a person to realize her vital goals is not (exactly) the same as for her to flourish in the Aristotelian sense. However, I am not certain that this immediately disqualifies my analysis of health. I think that it is at least equally difficult to argue that health, as a kind of ability, or even acting on this ability when realizing one's vital goals, should be tantamount to Aristotelian flourishing. For one thing that would make health, or healthy activity, equivalent to a certain degree of dignified life. I think there is a common sense distinction between health and dignified life and I think that the philosophical analysis of these notions should mirror this fact. 


\section{Concluding words}

I am grateful to Venkatapuram for his interesting comparison between Nussbaum's theory of capabilities and my own theory of health. Venkatapuram's critical remarks have given me the opportunity to place my own ideas in the context of the capability version of welfare theory. I now see much clearer where the similarities and differences between the two approaches are. Although I find some of Venkatapuram's remarks to be based on a misunderstanding of my writings, others have as their origin a different, and highly legitimate, basic attitude to the analysis of human welfare. I am therefore looking forward to further fruitful discussions on this issue.

\section{References}

Nordenfelt, L. (1995): On the Nature of Health: An Action-Theoretic Approach, Second, revised, edition, Kluwer Academic Publishers, Dordrecht.

Nordenfelt, L. (2000): Action, Ability and Health: Essays in the Philosophy of Health and Welfare, Kluwer Academic Publishers, Dordrecht.

Nordenfelt, L. (2007): The Concepts of Health and Illness Revisited, Medicine Health Care and Philosophy, 10: 5-10.

Nordenfelt, L. (ed.) (2009): Dignity in Care for Older People, Wiley-Blackwell, Oxford. Nussbaum, M.C. (2006): Frontiers of Justice: Disability, Nationality, Species Membership, The Belnap Press of Harvard University Press, Cambridge.

Nussbaum, M.C. (2011): Creating Capabilities: The Human Development Approach, The Belnap Press of Harvard University Press, Cambridge.

Schramme, T. (2007): A Qualified Defence of a Naturalist Theory of Health, Medicine, Health Care and Philosophy, 10: 11-17.

Venkatapuram, S. (2011): Health, Vital Goals, Capabilities, this volume. 